\title{
NeuroRegulation
}

\section{A Research Analysis of Neurofeedback Protocols for PTSD and Alcoholism}

\author{
Gordon Michael Russo ${ }^{1,2^{*}}$, and D. Allen Novian ${ }^{1,2}$ \\ ${ }^{1}$ The University of Texas at San Antonio, Texas, USA \\ ${ }^{2}$ The Novian Counseling \& Consulting Inc., San Antonio, Texas, USA
}

*Address correspondence to: Gordon Michael Russo, 12042 Blanco Rd., Suite 130, San Antonio, TX 78216; Email: michaelrusso@embarqmail.com

Copyright: (C) 2014 Russo and Novian. This is an Open Access article distributed under the terms of the Creative Commons Attribution License (CC-BY).

\begin{abstract}
A comparative literature research study was conducted and consisted of an analysis of two articles. Study one, by Peniston and Kulkosky, looks at Vietnam veterans with PTSD and the use of neurofeedback as a treatment modality. Study two, by Scott, Kaiser, Othmer, and Sideroff, examines the effect of neurofeedback as an additional therapeutic modality for people with chemical addictions. Both studies employ use of similar neurofeedback training methods.
\end{abstract}

Keywords: neurofeedback protocols

In the first study, the Minnesota Multiphasic Personality Inventory (MMPI) was used to assess personality changes in Vietnam combat veterans with posttraumatic stress disorder (PTSD) after either traditional medical treatment (TC) or alpha-theta BWT (brainwave neurofeedback therapy; Peniston \& Kulkosky, 1991). The subjects were selected based on the following criteria:

The subjects were randomly selected from a population of Vietnam veterans who were in combat situations and were evaluated for treatment of chronic combatrelated PTSD at Fort Lyon VA Medical Center; Diagnosis of combat-related PTSD as defined by DSM-III manual; No evidence of hallucinations or delusions; No known organic dysfunction; and Frequent recurring combat-related nightmares/flashbacks that were anxiety-evoking events (Peniston \& Kulkosky, 1991).

All subjects were given a brief introduction to EEG brainwave biofeedback and were told how to interpret the feedback sounds (i.e., Theta 2-7 Hz, Alpha 8-13 Hz, and Beta 15-18 Hz; Peniston \& Kulkosky, 1991). This meant that if brainwave activity was maintained a frequency higher than the threshold for alpha, beta, and theta, then the patient was rewarded with an auditory feedback tone and a visual reward consisting of a percentage counter that included the percentage above threshold. The subjects' earlobes and the inion were then 
cleaned with alcohol prior to attaching the electrodes. Each alpha-theta training session began with the subjects closing their eyes and sitting in a chair (Peniston \& Kulkosky, 1991; Scott et al., 2005). Two ear clip electrodes were attached and the active electrode was referenced to the left earlobe (A1), with the ground on the right earlobe (A2; Peniston \& Kulkosky, 1991; Scott, Kaiser, Othmer, \& Sideroff 2005). Omni Prep was then used as a conductive medium to fill the electrode cups and in the preparation of the electrode scalp site (Peniston \& Kulkosky, 1991).

The EEG system detected information in raw EEG by using three active band-pass filters. Filters consisted of tuning for Alpha, Beta, and Theta with $71 \mathrm{~dB}$ per octave attenuation rates (Peniston \& Kulkosky, 1991). Resulting information was fed back to the trainee virtually instantaneously with the conceptual understanding that changes in the feedback signal indicate whether or not the trainee's brain activity was within the designated range (ISNR, 2010).

Only BWT subjects received eight 30-minute sessions of pre-training in temperature biofeedback-assisted autogenic training and thirty 30-min BWT sessions (Peniston \& Kulkosky, 1991). It is believed that temperature training stimulates the production of the "theta state" (Peniston \& Kulkosky, 1991). The subjects were taught temperature biofeedback-assisted autogenic training due to the belief of its production of the "theta state" (Hall, 1977). All subjects, despite category of placement, agreed to participate in the study with the hope of alleviating symptoms associated with their combat-related PTSD (i.e., recurring nightmares and flashbacks, chronic states of anxiety, depression, vivid reexperiencing of traumatic combat events, etc.; Peniston \& Kulkosky, 1991).

To show the clinical affect on the subjects' life the following example is provided. This was not an isolated case and many of the Vietnam theater veterans' experienced repressed combat anxiety-provoking events during session: an episode of a repressed combat anxiety-evoking event concerned a patient who had nightmares of survivor guilt feelings. When the patient had a flashback at work, he became violent, destroying property, and he often had to be apprehended or sedated. While undergoing BWNT (Brain Wave Neurofeedback Therapy), the patient re-experienced a repressed combat anxiety-evoking when he was out in the bush with his buddy on patrol duty. His buddy was wounded and he hid him in the brush along the trail and returned to the helicopter for assistance. It was getting dark and the helicopter crewmen were apprehensive about remaining in the Viet Cong area after dark. The patient was told that his buddy would be all right until morning and they would return to get him in the early morning hours. The following morning the helicopter crew and patient returned to the area where his body was hidden. The patient saw his buddy nude, hanging by his feet from a tree, his body mutilated. The patient has felt survival guilt feelings for not going back to get his wounded buddy that evening and/or staying with him (Peniston \& Kulkosky, 1991).

BWNT appeared to allow those repressed Vietnam combat-related and anxiety-provoking events to become conscious by reliving them emotionally through hypnagogic imagery (Peniston \& Kulkosky, 1991). After one week of daily practice of BWT, the drug dosage (tricyclic antidepressants, antipsychotics, anxiolytics) for BWT subjects ( $n=14)$ and TC subjects $(n=13)$ was gradually reduced at their request (Peniston \& Kulkosky, 1991). 
An extension of the Peniston study was done in which 121 volunteers were chosen based on their primary drug of choice reported at admission: $31 \%$ heroin, $28 \%$ crack cocaine, $26 \%$ methamphetamine, $6 \%$ alcohol, and $9 \%$ other controlled substances; $94 \%$ were multipledrug users (Scott et al., 2005). If the subject was determined to have any other diagnosis or disorder based on the DSM-IV, they were unable to participate in the study (Scott et al., 2005).

In Phase I, experimental subjects underwent 10-20 sessions of Beta-SMR EEG biofeedback in which operant conditioning was used to augment either $15-18 \mathrm{~Hz}$ (beta) or 12-15 Hz (SMR) EEG activity. At the same time, training attenuated elevated activity in the 2-7 Hz (Theta) and 22-30 Hz (high Beta) ranges (Scott et al., 2005).

After ten Beta-SMR EEG biofeedback sessions, participants were reassessed with the TOVA, which is a neurophysiological measurement of attention (Scott et al., 2005). The experimental groups' TOVA results normalized after a mean of 13 sessions, meaning that they scored 85 or above (Scott et al., 2005).

In Phase II, subjects underwent 30 sessions of Alpha-Theta training. The frequency range for Alpha was $8-11 \mathrm{~Hz}$ and for Theta it was 5-8 Hz. The initial sessions were used to train down Alpha levels that were above $12 \mu \mathrm{V}$ (peak to peak), while augmenting Theta, until there was "crossover." This was defined as the point at which the Alpha amplitude drops below the level of Theta...Before crossover was achieved EEG activity in the range of $15-30 \mathrm{~Hz}$ was inhibited to reduce muscle tension (Scott et al., 2005).

Beta-SMR training had previously been shown to be effective in remediating attentional and cognitive deficits (Scott et al., 2005). The experimental group exhibited significant $(p<0.005)$ improvement compared with the changes in the control subjects on the following MMPI scales: Hs (Hypochondriasis), $F(1,81)=14.087$; D (Depression), $F(1,81)=48.129$; Hy (Conversion Hysteria), $F(1,81)=32.682$; Sc (Schizophrenia), $F(1,81)=15.241$; and Si (Social Introversion) scales, $F(1,81)=24.647$ (Scott et al., 2005). Furthermore, treating therapists reported that they noticed experimental subjects appeared more cooperative and more attentive as EEG biofeedback progressed (Scott et al., 2005).

In conclusion, with the employment of neurofeedback, both studies showed a significantly positive effect. The subjects, despite study type, showed an increase in quality of life following treatment. Both studies also showed how neurofeedback gave the clients the coping skills to decrease medication consumption and eliminate illegal drug usage. The feedback provided was created in "real-time" by the software and was non-invasive (ISNR, 2010). Due to this aspect, this was not an actual treatment in terms of non-organic input to the client; however, it was a learning method similar to that of traditional counseling therapeutic coping mechanisms. 


\section{References}

Hall, M. P. (1977). Theta training: Imagery and creativity. In E. E. Green \& A. M. Green (Eds.), Beyond biofeedback. San Francisco, CA: Delacorte Press.

International Society for Neurofeedback and Research. (2010). Learn about neurofeedback. Retrieved from http://www.isnr.org/neurofeedback-info/learn-more-aboutneurofeedback.cfm

Peniston, E. G., \& Kulkosky, P. J. (1991). Alpha-theta brainwave neuro-feedback for Vietnam veterans with combat-related post-traumatic stress disorder. Medical Psychotherapy, 4, 47-60.

Scott, W. C., Kaiser, D., Othmer, S., \& Sideroff, S. I. (2005). Effects of an EEG biofeedback protocol on a mixed substance abusing population. American Journal of Drug \& Alcohol Abuse, 31(3), 455-469. http://dx.doi.org/:10.1081/ADA-200056807 